\title{
The Optimal Stopping Time for Selling an Asset When It Is Uncertain Whether the Price Process Is Increasing or Decreasing When the Horizon Is Infinite
}

\author{
Nguyen Khac Minh', Nguyen Thanh Trung2, Pham Van Khanh1 \\ ${ }^{1}$ Thang Long Institute of Mathematics and Applied Science, Hanoi, Vietnam \\ ${ }^{2}$ Faculty of Fundamental Science, Military Academy of Logistics, Hanoi, Vietnam \\ Email: khacminh@gmail.com, nguyenthanhtrung.hvhc@gmail.com,van_khanh1178@yahoo.com
}

How to cite this paper: Minh, N.K., Trung, N.T. and Khanh, P.V. (2018) The Optimal Stopping Time for Selling an Asset When It Is Uncertain Whether the Price Process Is Increasing or Decreasing When the Horizon Is Infinite. American Journal of Operations Research, 8, 82-91.

https://doi.org/10.4236/ajor.2018.82007

Received: January 16, 2018

Accepted: March 6, 2018

Published: March 9, 2018

Copyright $\odot 2018$ by authors and Scientific Research Publishing Inc. This work is licensed under the Creative Commons Attribution International License (CC BY 4.0).

http://creativecommons.org/licenses/by/4.0/

\begin{abstract}
Assume that we want to shell an asset with unknown drift but known that the drift is a two value random variable, and the initial distribution can be estimated. As time goes by, this distribution is updated and base on the probability of the drift takes the small one gives us the stopping rule. Research results show that the optimal strategy to sell the asset is if the initial probability that the drift receives a small value greater than a certain threshold then liquidates the asset immediately, otherwise the asset holder will wait until the probability of the drift receives a small value passing a certain threshold, it is the optimal time to liquidate the asset.
\end{abstract}

\section{Keywords}

Optimal Stopping Time, Incomplete Information, Probability Process

\section{Introduction}

This paper considers the problem of optimal timing for selling of an asset under incomplete information about its drift. The asset price is assumed to follow a geometric Brownian motion $X$ with unknown drift, and an agent who decides to sell at time $t$ for which the expected value of the discounted asset price is maximized. Of course, when information is completed, the corresponding optimal liquidation problem is trivial. Indeed, if the drift of the asset price is larger than the interest rate, then on average the asset price grows faster than money in a risk-free bank account, and the agent should keep the asset as long as possible. 
Similarly, if the drift is smaller than the interest rate, it implies that the agent should liquidate the asset immediately and instead deposit the money in the bank.

In this paper we consider the incomplete information problem by modelling the drift as a random variable which takes two values. Then we reduce the two-dimensional problem into a one-dimensional optimal stopping problem.

There are many studies that have used various methods to solve the optimal stopping time problem. For instance, the authors in [1] gave the general theory of optimal stopping time and considered a set of optimal stopping time problems in areas such as mathematical statistics, mathematical finance, and financial engineering.

The optimal stopping time problem with deterministic drift is considered in [2] in various cases. To get the estimation of the parameters we use theory in [3] and [4] and use these estimated parameters to solve realistic optimal stopping problem. This paper is a continuation of the study in the paper [5] [6] and [7]. However, the problem is considered in the case that the last time is infinite so the method and the result are different from the previous results.

\section{The Problem and Its Solution}

We assume that the asset price process $X_{t}$ is modeled by a geometric Brownian motion (see [1]) as follows

$$
\mathrm{d} X_{t}=\mu X_{t} \mathrm{~d} t+\sigma X_{t} \mathrm{~d} W_{t}, t \geq 0
$$

where $\left\{W_{t}\right\}$ is a standard Brownian motion and this process is assumed to be independent of $\mu$ on a probability space $(\Omega, F, P)$. We also assume that the drift $\mu$ is a random variable which can take two values $\mu_{2}$ and $\mu_{1}$ satisfying the condition $\mu_{1}<r<\mu_{2}$, where $r \geq 0$ is constant interest rate, and $X_{0}$ is initial price. Assuming the property owner wants to sell his property but does not know the rate of increase of the price is $\mu_{1}$ or $\mu_{2}$ and he only knows that at the initial time the probability distribution of the events $\left\{\mu=\mu_{1}\right\}$ and $\left\{\mu=\mu_{2}\right\}$ as follows (Table 1).

The purpose of the property holder is to sell it for maximum expected returns and the purpose of this problem is the same. Mathematically we denote $\left\{\mathcal{F}_{t}^{\mathcal{X}}\right\}_{t \in[0 ; \infty)}$ be the $\sigma$-field generated by the process $X$ and property holders the choose $\mathcal{F}^{X}$-stopping time $\tau$ with $t \in[0 ; \infty)$ such that the supremum

$$
V=\sup _{\tau \in \mathcal{T}} E\left[\mathrm{e}^{-r \tau} X_{\tau}\right]
$$

is achieved at a certain stopping time.

For $t \geq 0$, let $\pi_{t}=P\left\{\mu=\mu_{1} \mid \mathcal{F}_{t}^{X}\right\}$ is the conditional probability that the

Table 1. The initial distribution of the drift.

\begin{tabular}{ccc}
\hline$\mu$ & $\mu_{1}$ & $\mu_{2}$ \\
Probability & $\pi$ & $1-\pi$ \\
\hline
\end{tabular}


drift receives small value at time $\mathrm{t}$ and therefore $1-\pi_{t}=P\left\{\mu=\mu_{2} \mid \mathcal{F}_{t}^{X}\right\}$.

From Theorems 7.12 and 9.1 in [3], the price process $X_{t}$ is modeled by the following stochastic differential equation

$$
\mathrm{d} X_{t}=\left[\mu_{1} \pi_{t}+\left(1-\pi_{t}\right) \mu_{2}\right] X_{t} \mathrm{~d} t+\sigma X_{t} \mathrm{~d} \bar{W}_{t}
$$

and the belief probability process $\pi_{t}$ satisfy equation

$$
\mathrm{d} \pi_{t}=-\omega \pi_{t}\left(1-\pi_{t}\right) \mathrm{d} \bar{W}_{t}
$$

where $\omega=\left(\mu_{2}-\mu_{1}\right) / \sigma$ and $\left(\bar{W}, F^{X}\right)$ is a $P$-Brownian motion defined by

$$
\mathrm{d} \bar{W}_{t}=\mathrm{d} W_{t}+\frac{\mu(t)-\left(1-\pi_{t}\right) \mu_{2}-\pi_{t} \mu_{1}}{\sigma} \mathrm{d} t .
$$

To reduce the dimension of the problem we define a new process $W$ by

$$
\mathrm{d} Z_{t}=-\left(\sigma+\omega \pi_{t}\right) \mathrm{d} t+\mathrm{d} \bar{W}_{t}
$$

and a new probability measure $Q$ with the Radon-Nikodym derivative as defined

$$
\begin{aligned}
\frac{\mathrm{d} Q}{\mathrm{~d} P} & =\mathrm{e}^{\left\{-\frac{1}{2} \int_{0}^{T}\left(\sigma+\omega \pi_{t}\right)^{2} \mathrm{dt}+\int_{0}^{T}\left(\sigma+\omega \pi_{t}\right) \mathrm{d} \bar{W}_{t}\right\}+\lambda t} \\
& =\mathrm{e}^{\left\{\frac{1}{2} \int_{0}^{T}\left(\sigma+\omega \pi_{t}\right)^{2} \mathrm{~d} t+\int_{0}^{T}\left(\sigma+\omega \pi_{t}\right) \mathrm{d} \mathrm{Z}_{t}\right\}+\lambda t}
\end{aligned}
$$

with respect to measure $P$ where $\lambda$ is chosen such that $\mu_{2}-\lambda-r<0$ and $-2\left(\mu_{2}-\lambda-r\right)-\omega^{2}>0$. Girsanov's Theorem so that $Z$ is a Brownian motion under measure $Q$.

We define a new process $\Phi_{t}=\frac{\pi_{t}}{1-\pi_{t}}$. Application of Ito's formula we obtain

$$
\begin{aligned}
\mathrm{d} \Phi_{t} & =\omega^{2} \pi_{t} \Phi_{t} \mathrm{~d} t+\frac{1}{\left(1-\pi_{t}\right)^{2}} \mathrm{~d} \pi_{t}=\omega^{2} \pi_{t} \Phi_{t} \mathrm{~d} t-\frac{\omega \pi_{t}\left(1-\pi_{t}\right)}{\left(1-\pi_{t}\right)^{2}} \mathrm{~d} \bar{W}_{t} \\
& =\omega^{2} \pi_{t} \Phi_{t} \mathrm{~d} t-\omega \Phi_{t} \mathrm{~d} \bar{W}_{t}=\omega^{2} \pi_{t} \Phi_{t} \mathrm{~d} t-\omega \Phi_{t}\left(\mathrm{~d} Z_{t}+\left(\omega \pi_{t}+\sigma\right) \mathrm{d} t\right) \\
& =-\sigma \omega \Phi_{t} \mathrm{~d} t-\omega \Phi_{t} \mathrm{~d} Z_{t} .
\end{aligned}
$$

Expressing of $X$ in terms of $Z$ gives

$$
\mathrm{d} X_{t}=\left(\sigma^{2}+\mu_{2}\right) X_{t} \mathrm{~d} t+\sigma X_{t} \mathrm{~d} Z_{t} .
$$

Then, under the $Q$ measure, both $X_{t}$ and $\Phi_{t}$ are geometric Brownian motions. Moreover, the $\sigma$-field generated by $Z$ and $X$ is coincided.

Now to build the calculations on the new measure we define the following process

$$
\theta_{t}=\mathrm{e}^{\left\{-\frac{1^{t}}{2}\left(\sigma+\omega \pi_{s}\right)^{2} \mathrm{ds}-\int_{0}^{t}\left(\sigma+\omega \pi_{s}\right) \mathrm{d} z_{s}\right\}-\lambda t} .
$$

Proposition 1. We have

$$
E\left[\mathrm{e}^{-r \tau} X_{\tau}\right]=\frac{X}{1+\phi} E^{Q}\left[\mathrm{e}^{\left(\mu_{2}-\lambda-r\right) \tau}\left(1+\Phi_{\tau}\right)\right]
$$

where $\tau$ is a stopping time adapts to the filter $\mathcal{F}^{X}$.

Proof: 
We have $\theta_{t}=\exp \left\{-\frac{1}{2} \int_{0}^{s}\left(\sigma+\omega \pi_{s}\right)^{2} \mathrm{~d} s-\int_{0}^{t}\left(\sigma+\omega \pi_{s}\right) \mathrm{d} Z_{s}\right\} \exp \{-\lambda t\}$ and therefore

$$
\frac{\mathrm{d} \theta_{t}}{\theta_{t}}=-\lambda \mathrm{d} t+\left(\omega \pi_{t}+\sigma\right) \mathrm{d} Z_{t}
$$

Consider the process

$$
K_{t}=\frac{x\left(1+\Phi_{t}\right) \mathrm{e}^{\left(\mu_{2}-\lambda\right) t}}{(1+\phi) X_{t}} .
$$

Using Ito's formula, we obtain

$$
\begin{aligned}
\mathrm{d} K_{t} & =\frac{\mathrm{e}^{\left(\mu_{t}-\lambda\right) t} x}{(1+\phi) X_{t}}\left(1+\Phi_{t}\right)\left[-\lambda \mathrm{d} t+\left(\omega \pi_{t}+\sigma\right) \mathrm{d} Z_{t}\right] \\
& =K_{t}\left[-\lambda \mathrm{d} t+\left(\omega \pi_{t}+\sigma\right) \mathrm{d} Z_{t}\right] .
\end{aligned}
$$

So $\theta_{t}=K_{t}$ almost sure. Thus we have:

$$
E\left[K_{t} X_{t} \mathrm{e}^{-r \tau}\right]=\frac{x}{1+\phi} E^{Q} \mathrm{e}^{\left(\mu_{l}-\lambda-r\right) \tau}\left(1+\Phi_{t}\right) .
$$

This proves the Proposition.

From the above Proposition the value function in the measure $Q$ given by

$$
V=\frac{x}{1+\phi} \sup _{\tau \in \mathcal{T}} E^{Q} \mathrm{e}^{\left(\mu_{l}-\lambda-r\right) \tau}\left(1+\Phi_{t}\right) .
$$

We see that larger $\Phi_{t}$ is the less likely that the gain will increase upon continuation. This suggests that there exists a point $B \in(0 ; \infty)$ such that the stopping time

$$
\tau_{B}:=\inf \left\{t \geq 0: \Phi_{t} \geq B\right\}
$$

is optimal in the problem (7).

By optimal stopping theory, the pair $(F, B)$ is the solution of the following free boundary problem

$$
\left\{\begin{array}{lr}
\mathcal{L} F+\left(\mu_{2}-\lambda-r\right) F=0 & 0<\phi<B \\
F(\phi)=1+\phi & \phi \geq B \\
F(B)=1+B & \text { (smooth condition) } \\
F^{\prime}(B)=1 & \\
F^{\prime}(1)=0 &
\end{array}\right.
$$

where $\mathcal{L} F=\frac{\omega^{2}}{2} \phi^{2} F^{\prime \prime}-\sigma \omega \phi F^{\prime}$ is infinitesimal operator and the condition $F^{\prime}(1)=0$ is added to define $F(\phi)$.

This follows that $F$ is the solution of the differential equation

$$
\frac{\omega^{2}}{2} \phi^{2} F^{\prime \prime}-\sigma \omega \phi F^{\prime}+\left(\mu_{2}-\lambda-r\right) F=0 .
$$


One may now recognize this differential equation as the Cauchy-Euler equation and it has characteristic equation:

$$
\frac{\omega^{2}}{2} x^{2}-\left(\sigma \omega+\frac{\omega^{2}}{2}\right) x+\mu_{2}-\lambda-r=0 .
$$

Let

$$
g(x)=\frac{\omega^{2}}{2} x^{2}-x\left(\sigma \omega+\frac{\omega^{2}}{2}\right)+\mu_{2}-\lambda-r .
$$

We find that

$$
\begin{gathered}
g(0)=\mu_{2}-\lambda-r<0 ; \\
g(1)=-\sigma \omega-\mu_{2}-\lambda-r=-\mu_{2}+\mu_{1}+\mu_{2}-\lambda-r=\mu_{1}-\lambda-r<0 .
\end{gathered}
$$

Therefore $g(x)$ has two solutions $x_{1}<0<1<x_{2}$ thus the general solution $F(\phi)$ can be written as

$$
F(\phi)=C_{1} \phi^{x_{1}}+C_{2} \phi^{x_{2}}, 0<\phi<B
$$

where $C_{1}$ and $C_{2}$ are constants which are determined later. The three conditions (9)-(11) can be used to determine $C_{1}, C_{2}$ and $B$ uniquely.

We have

$$
F^{\prime}(\phi)=C_{1} x_{1} \phi^{x_{1}-1}+C_{2} x_{2} \phi^{x_{2}-1}
$$

The condition $F^{\prime}(1)=0$ gives

$$
C_{1} x_{1}+C_{2} x_{2}=0
$$

and the condition $F^{\prime}(B)=1$ gives us $C_{1} x_{1} B^{x_{1}-1}+C_{2} x_{2} B^{x_{2}-1}=1$.

This follows

$$
\left\{\begin{array}{l}
C_{1}=\frac{1}{x_{1} B^{x_{1}-1}-x_{1} B^{x_{2}-1}} \\
C_{2}=\frac{-1}{x_{2} B^{x_{1}-1}-x_{2} B^{x_{2}-1}}
\end{array}\right.
$$

The condition $F(B)=1+B$ is equivalent to $C_{1} B^{x_{1}}+C_{2} B^{x_{2}}=1+B$.

Substitute $C_{1}$ and $C_{2}$ from (16) into above equation we have

$$
\frac{B^{x_{1}}}{x_{1} B^{x_{1}-1}-x_{1} B^{x_{2}-1}}-\frac{B^{x_{2}}}{x_{2} B^{x_{1}-1}-x_{2} B^{x_{2}-1}}=1+B .
$$

We have equation to determine $B$ as follow

$$
x_{1} x_{2} B^{x_{1}-1}-x_{1} x_{2} B^{x_{2}-1}+x_{2}\left(x_{1}-1\right) B^{x_{1}}+x_{1}\left(1-x_{2}\right) B^{x_{2}}=0 .
$$

Theorem 2.1. The equation (16) has unique solution $B \in(1 ; \infty)$.

Proof:

Consider function $h(t):=x_{1} x_{2}-x_{1} x_{2} t^{x_{2}-x_{1}}+x_{2}\left(x_{1}-1\right) t+x_{1}\left(1-x_{2}\right) t^{x_{2}-x_{1}+1}$ in $(0 ; \infty)$. We find that 


$$
h(1)=-x_{2}+x_{1}<0
$$

and $\lim _{t \rightarrow \infty} h(t)>0$ since $x_{1}\left(1-x_{2}\right)>0$. So equation $h(t)=0$ has a solution $B$ in $(1 ;+\infty)$.

We see that

$$
\begin{aligned}
h^{\prime}(t)= & x_{1}\left(1-x_{2}\right)\left(x_{2}-x_{1}+1\right) t^{x_{2}-x_{1}}+x_{2}\left(x_{1}-1\right)-x_{1} x_{2}\left(x_{2}-x_{1}\right) t^{x_{2}-x_{1}-1} \\
h^{\prime \prime}(t)= & x_{1}\left(1-x_{2}\right)\left(x_{2}-x_{1}+1\right)\left(x_{2}-x_{1}\right) t^{x_{2}-x_{1}-1} \\
& -x_{1} x_{2}\left(x_{2}-x_{1}\right)\left(x_{2}-x_{1}-1\right) t^{x_{2}-x_{1}-2}>0 .
\end{aligned}
$$

This follows

$$
\begin{aligned}
h^{\prime}(t) & \geq h^{\prime}(1)=-x_{1} x_{2}\left(x_{2}-x_{1}\right)+x_{2}\left(x_{1}-1\right)+x_{1}\left(1-x_{2}\right)\left(x_{2}-x_{1}+1\right) \\
& =\left(x_{2}-x_{1}\right)\left(-x_{1}\left(x_{2}-1\right)-x_{1} x_{2}-1\right) .
\end{aligned}
$$

We have

$$
\begin{aligned}
& \left(x_{2}-x_{1}\right)>0 ; \quad-x_{1}\left(x_{2}-1\right)>0 ; \\
& -x_{1} x_{2}-1=\frac{-2\left(\mu_{2}-\lambda-r\right)}{\omega^{2}}-1=\frac{-2\left(\mu_{2}-\lambda-r\right)-\omega^{2}}{\omega^{2}}>0
\end{aligned}
$$

by choosing $\lambda$ and therefore $h^{\prime}(t) \geq h^{\prime}(1)>0$.

Thus $h(t)$ is increasing function in $(1 ;+\infty)$. This implies there exists unique $B$ satisfies the problem.

Theorem 2.2.

$$
F(\phi)=\left\{\begin{array}{lc}
\frac{1+B}{C_{1} B^{x_{1}}+C_{2} B^{x_{2}}}\left(C_{1} \phi^{x_{1}}+C_{2} \phi^{x_{2}}\right), & 0<\phi<B \\
1+\phi, & \phi \geq B
\end{array}\right.
$$

with $B>1$ is unique solution off the following equation

$$
x_{1} x_{2}-x_{1} x_{2} B^{x_{2}-1}+x_{2}\left(x_{1}-1\right) B+x_{1}\left(1-x_{2}\right) B^{x_{2}-x_{1}+1}=0
$$

$C_{1}$ and $C_{2}$ defined in (16) and stopping time $\tau_{B}:=\inf \left\{t \geq 0: \Phi_{t} \geq B\right\}$ is optimal of $(7)$.

Proof:

Let $B$ be the unique solution to (17), and define function $G$ by

$$
G(\phi)=\left\{\begin{array}{lr}
\frac{1+B}{C_{1} B^{x_{1}}+C_{2} B^{x_{2}}}\left(C_{1} \phi^{x_{1}}+C_{2} \phi^{x_{2}}\right), & <\phi<B \\
1+\phi, & \phi \geq B
\end{array} .\right.
$$

Then we have $G(\phi) \geq 1+\phi$, and $G(\phi)>1+\phi$ if and only if $\phi<B$.

The process $Y_{t}=\mathrm{e}^{\left(\mu_{2}-\lambda-r\right) t} G\left(\Phi_{t}\right)$ satisfies the following stochastic differential equation

$$
\mathrm{d} Y_{t}=\mathrm{e}^{\left(\mu_{2}-\lambda-r\right) t}\left(\mu_{2}-\lambda-r+\left(\mu_{1}-\lambda-r\right) \Phi_{t}\right) 1_{\left\{\Phi_{t}>B\right\}} \mathrm{d} t-\mathrm{e}^{\left(\mu_{2}-\lambda-r\right) t} \omega \Phi_{t} G^{\prime}\left(\Phi_{t}\right) \mathrm{d} Z_{t} .
$$

Assume that the solution $B$ of (17) satisfies the condition $B \geq 0$. Then the drift of $Y$ is always negative, and therefore $Y$ is a super-martingale and $Y_{t \wedge \tau_{B}}$ is martingale.

Let $\tau$ is a stopping time. We have inequality 


$$
\mathbb{E}_{\phi} \mathrm{e}^{\left(\mu_{2}-\lambda-r\right) \tau}\left(1+\Phi_{t}\right) \leq \mathbb{E}_{\phi} Y_{\tau} \leq \mathbb{E}_{\phi} Y_{0}=G(\phi)
$$

This follows $F(\phi) \leq G(\phi)$.

And if $\tau=\tau_{B}$, we see that the inequalities in (18) are equalities.

Therefore,

$$
F(\phi)=\sup _{\tau} \mathbb{E}_{\phi}^{\mathbb{Q}} Y_{\tau} \geq \mathbb{E}_{\phi}^{\mathbb{Q}} Y_{\tau_{B}}=G(\phi) .
$$

It follows that $F(\phi)=G(\phi)$.

Corollary 2.2. Let $\phi=\frac{\pi}{1-\pi}$. The value function is given by:

$$
V=\left\{\begin{array}{lc}
x \frac{(1+B)}{C_{1} B^{x_{1}}+C_{2} B^{x_{2}}} \frac{\left(C_{1} \phi^{x_{1}}+C_{2} \phi^{x_{2}}\right)}{1+\phi}, & 0<\phi<B \\
x, & \phi \geq B
\end{array}\right.
$$

Moreover, stopping time $\tau_{B}:=\inf \left\{t \geq 0: \pi_{t} \geq \frac{B}{1+B}\right\}$ is optimal of problem (1).

Theorem 2.3. Value function $V$ is decreasing in $\pi$.

Proof:

When $\phi \geq B \Rightarrow \frac{\partial V}{\partial \phi}=0$. If $\phi \leq B$, we have

$$
\begin{aligned}
\frac{\partial V}{\partial \phi} & =x \frac{(1+B)}{C_{1} B^{x_{1}}+C_{2} B^{x_{2}}}\left(\frac{C_{1} \phi^{x_{1}}+C_{2} \phi^{x_{2}}}{1+\phi}\right)^{\prime} \\
& =x \frac{(1+B)}{C_{1} B^{x_{1}}+C_{2} B^{x_{2}}} C_{1} \frac{x_{1} x_{2} \phi^{x_{1}-1}-x_{1} x_{2} \phi^{x_{2}-1}+x_{2}\left(x_{1}-1\right) \phi^{x_{1}}+x_{1}\left(1-x_{2}\right) \phi^{x_{2}}}{(1+\phi)^{2}} \\
& =C_{1} x \frac{(1+B)}{C_{1} B^{x_{1}}+C_{2} B^{x_{2}}} \phi^{x_{1}-1} \frac{x_{1} x_{2}-x_{1} x_{2} \phi^{x_{2}-x_{1}}+x_{2}\left(x_{1}-1\right) \phi+x_{1}\left(1-x_{2}\right) \phi^{x_{2}-x_{1}+1}}{(1+\phi)^{2}} \\
& \leq 0
\end{aligned}
$$

If $\phi \geq 1$ the function

$$
h(\phi):=x_{1}\left(1-x_{2}\right) \phi^{x_{2}-x_{1}+1} x_{1} x_{2}-x_{1} x_{2} \phi^{x_{2}-x_{1}}+x_{2}\left(x_{1}-1\right) \phi
$$

is increasing function so $1 \leq \phi<B$ follows $h(\phi) \leq 0$ and if $0<\phi<1$ we have

$$
\begin{aligned}
h(\phi) & :=x_{1}\left(1-x_{2}\right) \phi^{x_{2}-x_{1}+1}+x_{1} x_{2}-x_{1} x_{2} \phi^{x_{2}-x_{1}}+x_{2}\left(x_{1}-1\right) \phi \\
& \leq x_{1}\left(1-x_{2}\right) \phi+x_{1} x_{2}-x_{1} x_{2}+x_{2}\left(x_{1}-1\right) \phi \\
& =\left[x_{2}\left(x_{1}-1\right)+x_{1}\left(1-x_{2}\right)\right] \phi=\left(x_{1}-x_{2}\right) \phi<0
\end{aligned}
$$

this gives that $V$ is decreasing in $\phi$ and $\phi$ is increasing function in $\pi$.

We complete the proof.

\section{Simulation}

In this section we simulate the price process, Posterior probability process and the threshold for selling the asset.

The parameters are 


$$
\begin{aligned}
& \mu_{1}=0.05 ; \mu_{2}=0.1 ; r=0.08 ; \lambda=0.05 ; \sigma=0.25 \\
& \omega=\left(\mu_{2}-\mu_{1}\right) / \sigma ; X(1)=3 ; \phi=1.1
\end{aligned}
$$

Let $x_{1}, x_{2}$ are the solutions of the following equation

$$
\frac{\omega^{2}}{2} x^{2}-\left(\sigma \omega+\frac{\omega^{2}}{2}\right) x+\mu_{2}-\lambda-r=0
$$

with the parameters are given in (19), equation (20) becomes:

$$
\frac{0.2^{2}}{2} x^{2}-\left(0.25 \times 0.2+\frac{0.2^{2}}{2}\right) x+0.1-0.05-0.08=0
$$

or $0.02 x^{2}-0.07 x-0.03=0$ and we have $x_{1}=-0.3860$ and $x_{2}=3.8860$.

And $B$ is the solution of

$$
x_{1} x_{2}-x_{1} x_{2} B^{x_{2}-1}+x_{2}\left(x_{1}-1\right) B+x_{1}\left(1-x_{2}\right) B^{x_{2}-x_{1}+1}=0 .
$$

We use Matlab software to solve Equation (21) and get $B=1.2798$.

In the Figure 1, we see that the posterior probability process cannot pass through the red threshold, so the holder of the property will not be able to sell it. However, since the time in simulation is finite, the property owner will sell the asset at the end that is $\tau_{B}=1$. And in the Figure 2 below the posterior probability shows that the fall in prices is very fast so the optimal stopping time is very close to the initial time.

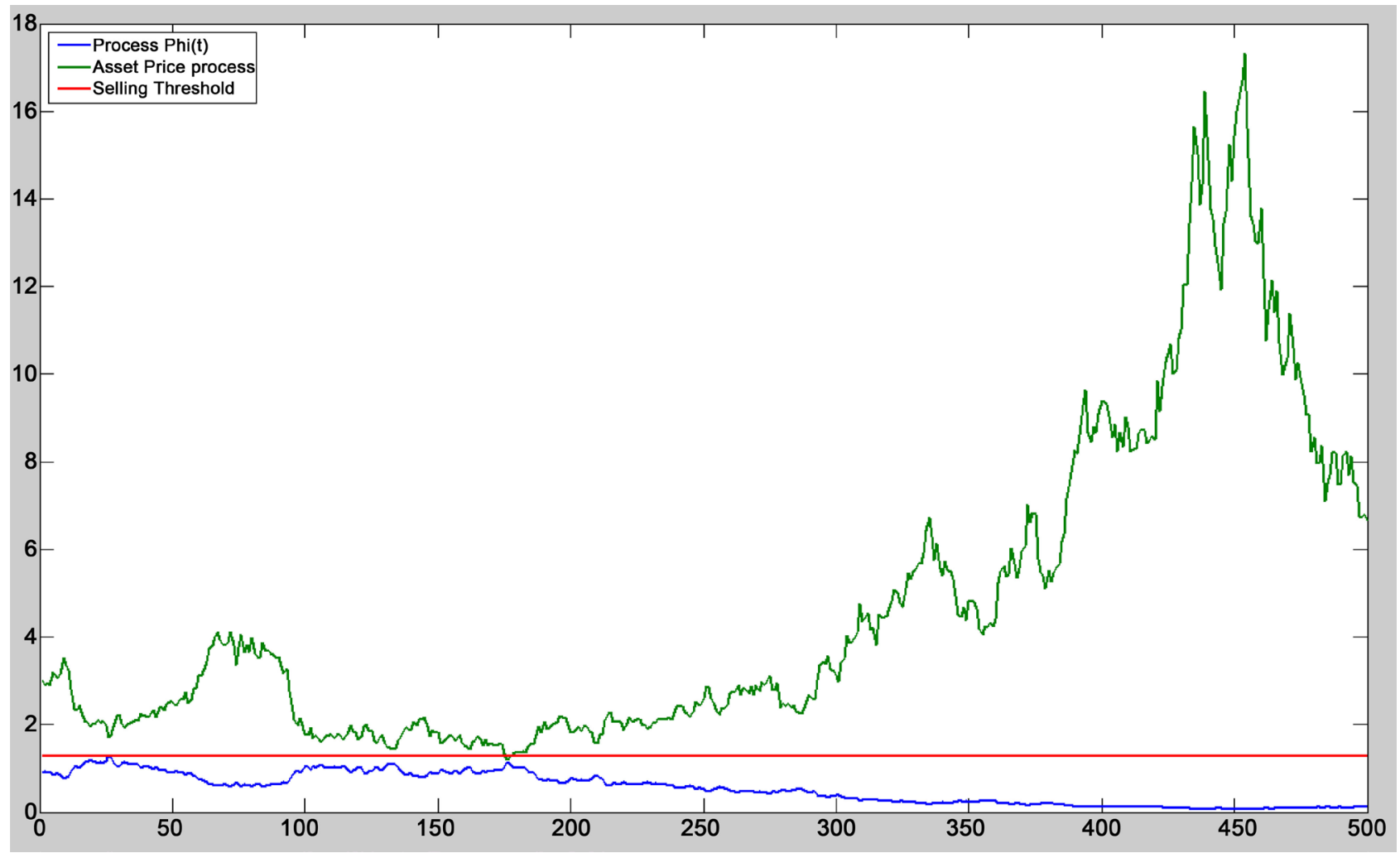

Figure 1. The stopping time and corresponding asset price in a simulation $\tau_{B}=1 ; \quad X\left(\tau_{B}\right)=6.98267216610124$. 


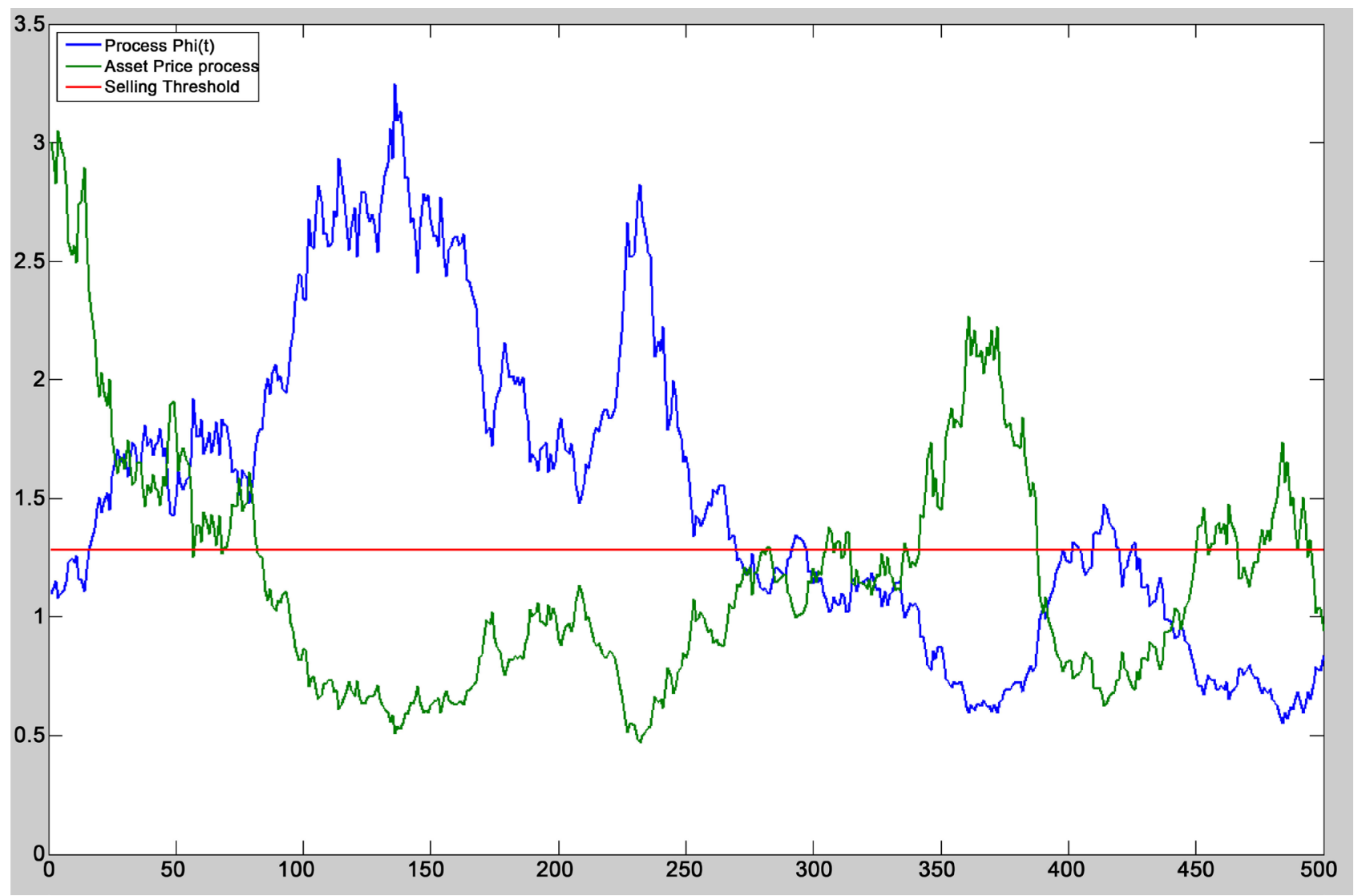

Figure 2. The stopping time and corresponding asset price in a simulation $\tau_{B}=0.030 ; X\left(\tau_{B}\right)=2.60306210985526$.

\section{Conclusion}

This paper studies the optimal strategy to liquidate an asset when it is uncertain whether the asset price is rising or falling (high or low drift). To solve the problem we have to change the initial measure to the new measure and under this measure the price process is martingale. We also have to solve a nonlinear equation to find the threshold of the probability that the drift receives the smaller value. The results show that the value function is decreasing in the initial probability that the drift is low. Simulation results are consistent with proven theory.

\section{References}

[1] Peskir, G. and Shiryaev, A.N. (2006) Optimal Stopping and Free-Boundary Problems (Lectures in Mathematics ETH Zürich). Birkhäuser, Basel.

[2] Shiryaev, A.N., Xu, Z. and Zhou, X.Y. (2008) Thou Shalt Buy and Hold. Quantitative Finance, 8, 765-776. https://doi.org/10.1080/14697680802563732

[3] Lipster, R.S. and Shiryaev, A.N. (2001) Statistics of Random Process: I. General Theory. Springer-Verlag, Berlin, Heidelberg.

[4] Shiryaev, A.N. (2008) Optimal Stopping Rules. Springer-Verlag, Berlin, Heidelberg.

[5] Khanh, P. (2012) Optimal Stopping Time for Holding an Asset. American Journal of Operations Research, 2, 527-535. https://doi.org/10.4236/ajor.2012.24062

[6] Khanh, P. (2014) Optimal Stopping Time to Buy an Asset When Growth Rate Is a 
Two-State Markov Chain. American Journal of Operations Research, 4, 132-141. https://doi.org/10.4236/ajor.2014.43013

[7] Van Khanh, P. (2015) When to Sell an Asset Where Its Drift Drops from a High Value to a Smaller One. American Journal of Operations Research, 5, 514-525. https://doi.org/10.4236/ajor.2015.56040 